\title{
Mortality risk in post-operative head and neck cancer patients during the SARS-Cov2 pandemic: early experiences
}

\author{
Justin M. Hintze $\mathrm{e}^{1,2,3}$ (C) Conall W. Fitzgerald ${ }^{1,2,3} \cdot$ Bronagh Lang $^{1,2,3} \cdot$ Paul Lennon $^{1,2} \cdot$ John B. Kinsella ${ }^{1,2}$
}

Received: 23 May 2020 / Accepted: 16 June 2020 / Published online: 22 June 2020

c) Springer-Verlag GmbH Germany, part of Springer Nature 2020

\begin{abstract}
Purpose The objective of this report is to outline our early experience with head and neck cancer patients in a tertiary referral center, during the SARS-Cov2 pandemic, and to describe the poor outcomes of patients who acquired the infection. Methods In this case series from a single-center, national tertiary referral center for head and neck cancer we describe three consecutive head and neck cancer patients who contracted SARS-Cov2 during their inpatient stay.

Results Of the three patients described in our case series that contracted SARS-Cov2, two patients died from SARS-Cov2 related illness.

Conclusion We have demonstrated the significant implications that SARS-Cov2 has on head and neck cancer patients, with 3 patients acquiring SARS-Cov2 in hospital, and 2 deaths in our that cohort. We propose a complete separation in the location of where these patients are being managed, and also dedicated non-SARS-Cov2 staff for their peri-operative management. Level of evidence IV.
\end{abstract}

Keywords Head and neck cancer $\cdot$ SARS-Cov2 $\cdot$ Mortality $\cdot$ Covid-19

\section{Introduction}

The SARS-Cov2 pandemic has major implications for the delivery of elective Otolaryngology-Head and Neck Surgery (ORL-HNS) oncology treatment internationally, with services in many jurisdictions coming largely to a standstill. ORL-HNS surgeons were noted to be in a high-risk group for transmission in reports from Wuhan, China, the UK and Italy [1-4]. Very high mortality rates have been reported in a wide range of post-operative elective surgery patients who were operated on in the prodromal phase of SARS-Cov2 [5].

Guidelines have been published for pre- and intra-operative management and protection to help reduce the risk of spread of SARS-Cov2 to patients and staff $[1,2,6]$. The post-operative implications for these high-risk patients are not yet clear.

Justin M. Hintze

hintzej@tcd.ie

1 Department of Otolaryngology, Head and Neck Surgery, St. James Hospital Dublin, Dublin, Ireland

2 Trinity College, University of Dublin, Dublin, Ireland

3 Royal College of Surgeons in Ireland, Dublin, Ireland
With this in mind, we describe our early experience with three post-operative head and neck cancer patients at our institution, as it relates to the SARS-Cov2 pandemic, and the repercussions for future surgical practices.

\section{Methods}

We describe our experience of three patients with advanced head and neck cancer that underwent treatment prior to the onset the SARS-Cov2 pandemic in Ireland, who subsequently contracted SARS-Cov2 during their post-operative hospital stay. Our institution is the largest acute hospital in Ireland and a national tertiary-referral center for head and neck. This research was granted institutional review board exemption from the Tallaght University Hospital-St. James Hospital Joint Research Ethics Committee. Informed consent was obtained from the patient directly where possible, or their next of kin in cases where the patient was deceased. 


\section{Results}

Patient 1 was admitted to our institution for definitive treatment of tracheal stomal seeding from a previously resected T4 squamous cell carcinoma (SCC) of the oral cavity. He underwent a total laryngectomy with partial tracheal resection, followed by reconstruction with a pectoralis major pedicled flap. Final histology revealed clear margins.

Table 1 Patient demographics

\begin{tabular}{llll}
\hline & Patient 1 & Patient 2 & Patient 3 \\
\hline Age & 56 & 68 & 62 \\
Primary site & Trachea & Larynx & Hypopharynx \\
Date of admission & $23 / 02 / 2020$ & $04 / 02 / 2020$ & $07 / 03 / 2020$ \\
Date of surgery & $09 / 03 / 2020$ & $25 / 02 / 2020$ & $10 / 03 / 2020$ \\
Type of surgery & Laryngectomy & Tracheotomy, ND & Tracheotomy \\
Date of SARS- & $07 / 04 / 2020$ & $17 / 4 / 2020$ & $16 / 04 / 2020$ \\
Cov2 & & & \\
Ferritin & 3137 & 1139 & 124 \\
D-Dimer & 6885 & 1161 & N/A \\
IL-6 & 144 & 56 & 4.8 \\
CD25 & $>5000$ & 3583 & 1770 \\
Date of death & $14 / 04 / 2020$ & $30 / 04 / 2020$ & \\
\hline
\end{tabular}

The patient's post-operative course was complicated by wound healing issues related to his prior radiation treatment, as well as an acute exacerbation of the chronic renal disease. This caused a prolonged inpatient stay. Otherwise, the patient remained clinically well, with no evidence of post-operative infections. On his 4th post-operative week, a patient in the same ward contracted SARS-Cov2, which prompted isolation and testing of our patient. He was also found to be SARS-Cov2 positive, with elevated SARS-Cov2 markers (Table 1). While the patient initially remained stable, his d-dimer and ferritin continued to climb, and his oxygen requirements escalated 6 days after testing positive for SARS-Cov2. He was commenced on hydroxychloroquine and broad-spectrum antibiotics. He continued to deteriorate both from a respiratory and renal point of view. He was transferred to the intensive care unit (ICU) for ventilatory support and continuous renal replacement therapy. He subsequently succumbed to respiratory complications from SARS-Cov2 7 days after diagnosis (Fig. 1).

Patient 2 presented with progressive hoarseness, dysphagia and weight loss secondary to a large obstructing supraglottic T4aN2bM0 SCC.

At the time of surgery, the tumor was found to be directly invading his right common carotid artery and was deemed unresectable, upstaging him to T4b disease. A tracheotomy was performed to protect his airway. Following repeat

25000

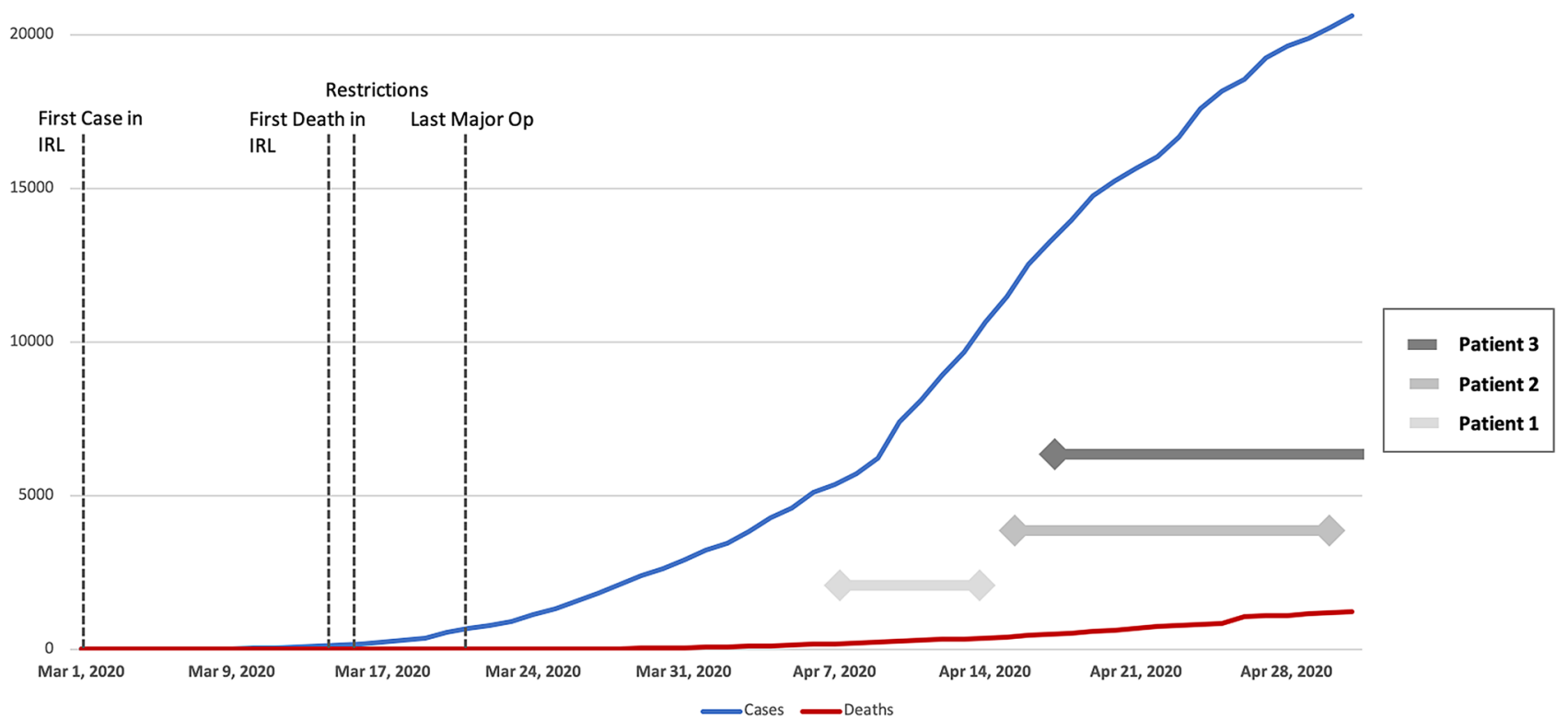

Fig. 1 Timeline including first cases and deaths in Ireland and when country-wide restrictions were implemented. IRL: Ireland, deaths: total number of deaths in Ireland, cases: total number of cases in Ireland 
discussion at the head and neck oncology MDT he commenced palliative radiotherapy (40 Gy/15\#) as an inpatient.

He developed respiratory symptoms during week two of radiotherapy treatment, with increased tracheal secretions and shortness of breath as well as tachycardia, reduced oxygen saturations and raised inflammatory markers. He was swabbed and tested positive for SARS-Cov2 the following day, with elevated SARS-Cov2 markers (Table 1). Unfortunately, he continued to deteriorate, his radiotherapy treatment was stopped and palliative care was initiated. His chest $\mathrm{x}$-ray demonstrated progressive SARS-Cov2 infection with diffuse airspace opacification throughout both lungs and he died 15 days following SARS-Cov2 diagnosis (Fig. 1).

Patient 3 was receiving full-dose concurrent chemo-radiotherapy for a T4b hypopharyngeal SCC with curative intent when he was admitted to hospital with new-onset stridor. Flexible nasolaryngoscopy revealed significant radiotherapy-related supraglottic edema that failed to settle with medical management with steroid and inhaled epinephrine. $\mathrm{He}$ underwent tracheotomy to protect his compromised airway, which was completed without complication. The patient subsequently resumed radiotherapy treatment as an inpatient, as he was unsuitable for immediate discharge. One month after admission to hospital he was found to be SARS-Cov2 positive, having shared a ward with patient 2 . His initial bloods were not significantly elevated (Table 1). As he had less than 1 week of radiation treatment remaining, it was decided to halt his treatment. He remains well without oxygen requirements and is planned for discharge in the coming week.

\section{Discussion}

The SARS-Cov2 pandemic has brought multiple challenges to clinical practice for ORL-HNS [1]. Strategies are emerging to overcome these difficulties during the pandemic, but concern remains for the high risk of mortality in patients contracting the infection in the postoperative period. A case series from Wuhan of 34 patients who underwent a range of elective surgeries during the incubation period of SARS-Cov2 were found to have very poor outcomes, with all patients developing SARS-Cov2 pneumonia shortly after surgery. Of those, $44 \%$ required admission to the ICU, and there was a $20.5 \%$ case fatality rate [5].

Furthermore, head and neck cancer patients are likely to have an increased risk of adverse outcomes from contracting SARS-Cov2 [2, 7]. They frequently have multiple comorbidities, are typically elderly, smokers with poor performance status. The current case-fatality rate from SARSCov2 for patients above age 70 is estimated to be between 8 and $22.5 \%[3,8]$. The presence of a tracheotomy or laryngectomy stoma is also thought to allow the easy transmission to and from the patient [9].
Each of our three patients was admitted to hospital before Ireland recorded its first SARS-Cov2 death on the 12th of March, and when the total number of cases in the country was less than 10 (Fig. 1). Furthermore, their procedures were carried out far in advance of the country-wide restrictions put into place on the 13th of March. However, head and neck cancer patients frequently require prolonged inpatient stay post-operatively, in particular those with a tracheotomy, advanced stage or prior radiation [10]. These patients are at increased risk of transmission during care and this risk is exacerbated by a lengthy hospital stay.

In response to the SARS-Cov2 pandemic, our institution implemented aggressive measures to help discharge patients from the hospital, isolate and protect existing inpatients, and create alternative pathways for prospective surgical candidates at other institutions, which are nonreceiving SARS-Cov2 hospitals. Five patients admitted or treated prior to the onset of wide-spread restrictions or SARS-Cov2 cases in Ireland, were unable to be promptly discharged, either due to medical or social reasons. Three of these patients contracted SARS-Cov2, despite routine SARS-Cov2 institutional precautions in keeping with existing best-practice international guidelines. Two of these patients subsequently died from complications of SARS-Cov2 infection.

Our experience suggests that patients undergoing major head and neck cancer surgery are at very high risk during the SARS-Cov2 pandemic. We recommend aggressive separation and precautions for this vulnerable patient cohort. We propose two potential strategies to achieve this. We suggest the nomination of a SARS-Cov2 free hospital as the center for head and neck surgery, or stringent separation at a hospital level into non-SARS-Cov2 and SARS-Cov2 "streams". In the latter scenario, patients would be cared for only by staff not exposed to SARSCov2 or high-risk patients. This is supported by evidence from the 2003 SARS outbreak, where it was shown that these patients should be cared for in entirely separate units, or even separate hospitals by designated health care workers [11-14]. Finally, stringent pre-operative testing and isolation of patients being considered for major head and neck surgery is also of utmost importance, as patients with prodromal SARS-Cov2 were also found to have very poor outcomes [5].

This is a small case series and thus has inherent limitations. However, other than the case series from Wuhan [5], there are as yet no further reports of postoperative surgical mortality in the SARS-Cov2 era. This is likely to be due to increased precautions in hospitals and decreased surgical activity across the globe. However, as countries 'emerge from the lockdown', it is likely that the risk to post-operative patients will become more apparent. 


\section{Conclusions}

Head and neck cancer patients are not only part of a vulnerable patient cohort, but also present unique challenges for post-operative care. Their prolonged inpatient stay and levels of input from numerous health care professionals puts them at significantly increased risk of nosocomial SARS-Cov2 infection. We have demonstrated the significant implications that SARS-Cov2 has on these patients, with 3 cases of acquired SARS-Cov2 in hospital and 2 deaths in our inpatient cohort.

We propose a complete separation in the location of where these patients are being cared for, and also dedicated non-SARS-Cov2 staff for their peri-operative management.

Author contributions All authors contributed equally to conception and design of the manuscript. All authors contributed to drafting of the article and approved the final and are accountable for all aspects of the work.

Funding Not applicable.

Availability of data and material Not applicable.

\section{Compliance with ethical standards}

Conflict of interest The author(s) declare that they have no competing interests.

Ethics approval This manuscript was given institutional review board exemption.

Consent to participate All patients, or next-of-kin if deceased, consented to participate.

Consent for publication All patients, or next-of-kin if deceased, consented for publication.

Code availability Not applicable.

\section{References}

1. Givi B, Schiff BA, Chinn SB et al (2020) Safety recommendations for evaluation and surgery of the head and neck during the COVID-19 pandemic. JAMA Otolaryngol Head Neck Surg. https ://doi.org/10.1001/jamaoto.2020.0780
2. Day AT, Sher DJ, Lee RC et al (2020) Head and neck oncology during the COVID-19 pandemic: reconsidering traditional treatment paradigms in light of new surgical and other multilevel risks. Oral Oncol 105:104684

3. Livingston E, Bucher K (2020) Coronavirus disease 2019 (COVID-19) in Italy. JAMA. 323(14):1335

4. Zhu W, Huang X, Zhao H, Jiang X (2020) A COVID-19 Patient who underwent endonasal endoscopic pituitary adenoma resection: a case report. Neurosurgery. https://doi.org/10.1093/neuros/ nyaa 147

5. Lei S, Jiang F, Su W et al (2020) Clinical characteristics and outcomes of patients undergoing surgeries during the incubation period of COVID-19 infection. EClinicalMedicine. https://doi. org/10.1016/j.eclinm.2020.100331

6. Sommer DD, Engels PT, Usaf C et al (2020) Recommendations from the CSO-HNS taskforce on performance of tracheotomy during the COVID-19 pandemic. J Otolaryngol Head Neck Surg. 49(1):23

7. Mehta V, Goel S, Kabarriti R et al (2020) Case fatality rate of cancer patients with COVID-19 in a New York Hospital system. Cancer Discov

8. Wu Z, McGoogan JM (2020) Characteristics of and important lessons from the coronavirus disease 2019 (COVID-19) outbreak in China: summary of a report of 72314 cases from the chinese center for disease control and prevention. JAMA. 323:1239-1242

9. Tran K, Cimon K, Severn M, Pessoa-Silva CL, Conly J (2012) Aerosol generating procedures and risk of transmission of acute respiratory infections to healthcare workers: a systematic review. PLoS One 7(4):e35797

10. McDevitt J, Cancela Mde C, Kelly M, Comber H, Sharp L (2016) Tracheostomy and infection prolong length of stay in hospital after surgery for head and neck cancer: a population based study. Oral Surg Oral Med Oral Pathol Oral Radiol. 121(1):22-28e21

11. Dwosh HA, Hong HH, Austgarden D, Herman S, Schabas R (2003) Identification and containment of an outbreak of SARS in a community hospital. CMAJ 168(11):1415-1420

12. Gamage B, Moore D, Copes R, Yassi A, Bryce E, Group BCIRPS (2005) Protecting health care workers from SARS and other respiratory pathogens: a review of the infection control literature. Am J Infect Control 33(2):114-121

13. Li Y, Huang X, Yu IT, Wong TW, Qian H (2005) Role of air distribution in SARS transmission during the largest nosocomial outbreak in Hong Kong. Indoor Air 15(2):83-95

14. Yen MY, Lin YE, Su IJ et al (2006) Using an integrated infection control strategy during outbreak control to minimize nosocomial infection of severe acute respiratory syndrome among healthcare workers. J Hosp Infect 62(2):195-199

Publisher's Note Springer Nature remains neutral with regard to jurisdictional claims in published maps and institutional affiliations. 